\title{
In vitro evaluation of new terpenoid derivatives against Leishmania infantum and Leishmania braziliensis
}

\author{
Inmaculada Ramírez-Macías', Clotilde Marín', Rachid Chahboun², Francisco Olmo, \\ Ibtisam Messouri ${ }^{2}$, Oscar Huertas ${ }^{1}$, María Jose Rosales ${ }^{1}$, Ramón Gutierrez-Sánchez ${ }^{3}$, \\ Enrique Alvarez-Manzaneda ${ }^{2}$, Manuel Sánchez-Moreno ${ }^{1 /+}$
}

\author{
${ }^{1}$ Department of Parasitology ${ }^{2}$ Department of Organic Chemistry, Institute of Biotechnology \\ ${ }^{3}$ Department of Statistics, University of Granada, Avda. Severo Ochoa s/n, E-18071 Granada, Spain
}

\begin{abstract}
The activity of five (1-5) abietane phenol derivatives against Leishmania infantum and Leishmania braziliensis was studied using promastigotes and axenic and intracellular amastigotes. Infectivity and cytotoxicity tests were performed with J774.2 macrophage cells using Glucantime as a reference drug. The mechanisms of action were analysed by performing metabolite excretion and transmission electron microscopy ultrastructural studies. Compounds 1-5 were more active and less toxic than Glucantime. The infection rates and mean number of parasites per cell observed in amastigote experiments showed that derivatives 2, 4 and 5 were the most effective against both $\mathrm{L}$. infantum and $\mathrm{L}$. braziliensis. The ultrastructural changes observed in the treated promastigote forms confirmed that the greatest cell damage was caused by the most active compound (4). Only compound 5 caused changes in the nature and amounts of catabolites excreted by the parasites, as measured by ${ }^{1} \mathrm{H}$ nuclear magnetic resonance spectroscopy. All of the assayed compounds were active against the two Leishmania species in vitro and were less toxic in mammalian cells than the reference drug.
\end{abstract}

Key words: abietane phenol compounds - Leishmania infantum - Leishmania braziliensis biological evaluation of activity - promastigote - amastigote forms

Leishmaniasis, which is widely considered to be a "neglected disease", is common in tropical and subtropical regions, including 22 countries in the New World and 66 in the Old World (who.int/leishmaniasis/). The etiological agents of this disease are different species of protozoa from the genus Leishmania, which are transmitted by flies (Diptera) from the genus Phlebotomus in the Old World and from Lutzomyia in the New World. These species cause cosmopolitan or endemic diseases and present serious public health problems and leishmaniasis is considered by the WHO to be one of the seven highest priority diseases that affect all continents.

Pentavalent antimonial compounds, such as sodium stibogluconate (Pentostam) and meglumine antimoniate (Glucantime), have been recommended as first-line drugs for the treatment of leishmaniasis for 50 years. The most frequent side effects of these compounds, including anorexia, vomiting, peripheral polyneuropathy and allergic dermopathy, are probably a result of oxidative or reductive damage to the host tissue. Thus, they are inextricably linked to the antiparasitic activity of the compounds (Momeni et al. 2002, Natera et al. 2007, Palumbo 2009). These effects make the development of new drugs

Financial support: Proyecto de Excelencia de la Junta de Andalucia Project (P07-FQM-03101), MEC (Spain) (CGL2008-03687-E/BOS) + Corresponding author: msanchem@ugr.es

Received 19 July 2011

Accepted 14 February 2012 for the treatment of leishmaniasis a highly relevant and challenging research goal (Docampo \& Moreno 1986, Cerecetto \& Gonzalez 2002, Palumbo 2009).

One method of drug discovery involves the investigation of natural products obtained from medicinal plants (Braña et al. 2005). Indeed, folk medicines are often valid sources of bioactive substances that are potentially useful in the treatment of many diseases, as demonstrated by the search for new medicinal agents for the treatment of trypanosomiasis, leishmaniasis and other diseases (Braña et al. 2005). In fact, a broad range of plant families and species contain active trypanocidal and leishmanicidal substances (Muhammad et al. 2002, Cui et al. 2003, Takahashi et al. 2004, González et al. 2005, Tasdemir et al. 2006, Corrêa et al. 2011). For example, diterpene resin acids, which are found in conifers, are known to be a potent defence against herbivores and pathogens (Martin et al. 2002). Likewise, the reported biological activities of natural abietane acids include antimicrobial, antiulcer, cardiovascular, antiallergic, filmogenic, surfactant and anti-feedant properties (San Feliciano et al. 1993). The interest in this type of terpenoid has increased in recent years and has been encouraged by the isolation of several compounds, mainly phenols and related derivatives, with remarkable biological activities (Marrero et al. 2002, Tan et al. 2002). Other significant oxidised abietane diterpenes have been shown to strongly inhibit various human tumours and oncogene-transformed cells (Son et al. 2005).

Our group has recently synthesised five novel abietane phenols (Alvarez-Manzaneda et al. 2007a, b, c). To determine the benefits of these compounds, we studied 
their in vitro antiproliferative activities against extra and intracellular forms of two species of Leishmania, Leishmania infantum and Leishmania braziliensis. The potential cytotoxicity of these compounds was also assessed using non-parasitised host cells to establish whether the in vitro activity of the metabolite is due to its general cytotoxic activity or whether it is selectively active against Leishmania (Luque et al. 2000). Furthermore, we performed nuclear magnetic resonance spectroscopy ( ${ }^{1} \mathrm{H}$ NMR) to determine the nature and percentages of the metabolites excreted by Leishmania and to clarify the potential of the compounds to inhibit the glycolytic pathway, which is the primary source of energy for the parasite. Finally, the effect of these compounds on the ultrastructure of Leishmania spp was monitored by transmission electron microscopy (TEM).

\section{MATERIALS AND METHODS}

Chemical compounds - Compound 1, the methyl ester of 12-hydroxydehydroabietic acid, has recently been described as a new natural product (Kinouchi et al. 2000) and was synthesised from commercial abietic acid (Alvarez-Manzaneda et al. 2007b) (Fig. 1). Compounds 2-4 were prepared from trans-communic acid (Alvarez-Manzaneda et al. 2007c), a labdane diterpene, that is very abundant in some species of Juniperus and Cupressus. Compound 3 is the methyl ester of lambertic
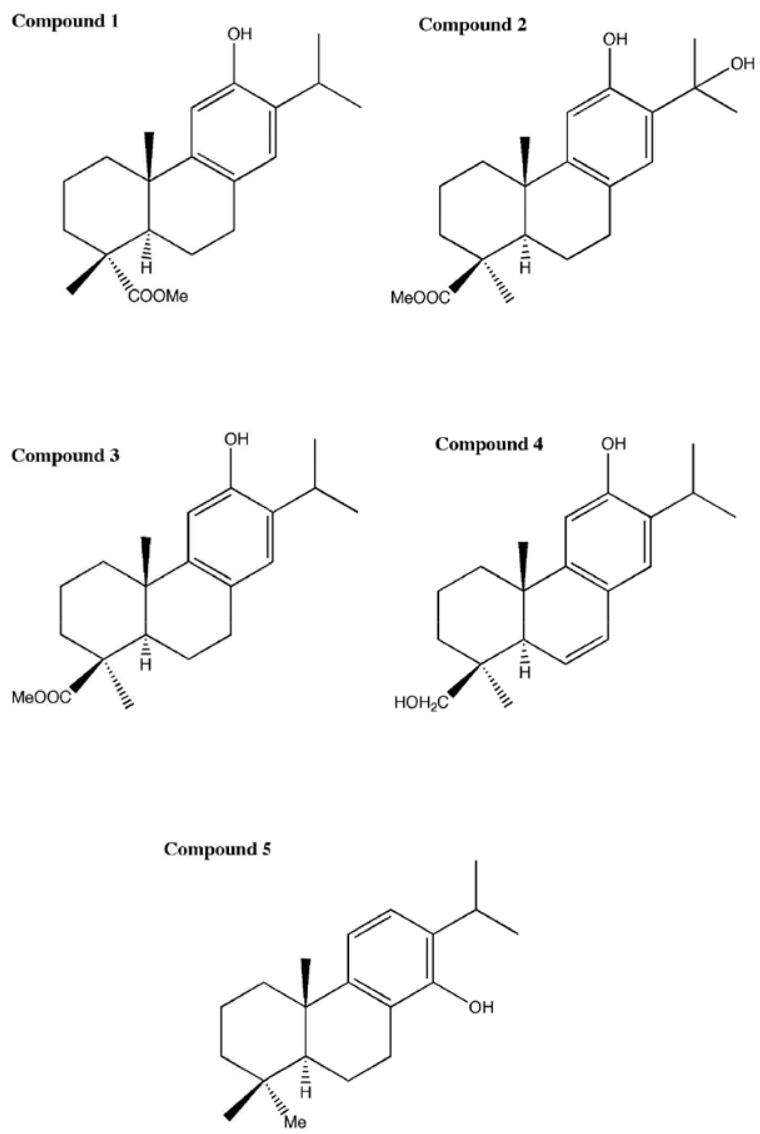

Fig. 1: chemical structures of the terpenoid derivatives. acid, which was isolated from Podocarpus lambertius, and compound 4, 6,7-dehydroabieta-8,11,13-trien-12,19-diol, named sugikurojin A, is a new diterpene that was recently isolated from Cryptomeria japonica. Compound 2, methyl-12,15-dihydroxyabieta-8,11,13-trien19 -oate, has not yet been found in nature. Compound 5, abieta-8,11,13-trien-14-ol, which was prepared from abietic acid (Alvarez-Manzaneda et al. 2007a), is an immediate precursor in the synthesis of the antileishmanial agent 12-deoxyroyleanone.

Parasite strain culture - L. infantum (MCAN/ ES/2001/UCM-10) and L. braziliensis (MHOM/ BR/1975/M2904) were cultivated in vitro in medium trypanosomes liquid (MTL) containing 10\% inactive foetal bovine serum (FBS) and were maintained in a standard air atmosphere at $28^{\circ} \mathrm{C}$ in Roux flasks (Corning, USA) with a surface area of $75 \mathrm{~cm}^{2}$, as described by González et al. (2005).

Cell culture and cytotoxicity tests - J774.2 macrophages (ECACC 91051511) were originally obtained from a tumour in a female BALB/c mouse in 1968. The cytotoxicity test for macrophages was performed as described by González et al. (2005). After $72 \mathrm{~h}$ of treatment, the cell viability was determined by flow cytometry. The cells were first incubated for $10 \mathrm{~min}$ with $100 \mu \mathrm{L} /$ well of propidium iodide solution $(100 \mathrm{mg} / \mathrm{mL})$ at $28^{\circ} \mathrm{C}$ in darkness. Subsequently, $100 \mu \mathrm{L} /$ well of fluorescein diacetate $(100 \mathrm{ng} / \mathrm{mL})$ was added and the cells were incubated under the same conditions. Finally, the cells were recovered by centrifugation at $400 \mathrm{~g}$ for $10 \mathrm{~min}$ and the precipitate was washed with phosphate-buffered saline (PBS). Flow cytometric analysis was performed with a FACSVantage flow cytometer (Becton Dickinson, San Agustin de Guadalix, Madrid, Spain). The percent viability was calculated in comparison to the control culture. The $\mathrm{IC}_{50}$ was calculated using linear regression analysis from the $\mathrm{Kc}$ values of the concentrations that were employed.

In vitro activity assay - Promastigote forms - The promastigote forms were collected in the exponential growth phase and distributed in culture trays (with 24 wells) to a final concentration of $5 \times 10^{4}$ parasites/well. The compounds were dissolved in MTL and dimethyl sulphoxide (Panreac, Barcelona, Spain) at a concentration of $0.01 \%$, after each compound was shown to be non-toxic and non-inhibitory of the growth of the parasites. The compounds were tested at final concentrations of 1-100 $\mu \mathrm{M}$. The effects of each compound against the promastigote forms were tested at $72 \mathrm{~h}$ using a Neubauer haemocytometric chamber. The antileishmanial effects were expressed as $\mathrm{IC}_{50}$ values, i.e., the concentration required to produce $50 \%$ inhibition, which were calculated by linear regression analysis of the Kc values of the tested concentrations.

Amastigote assay - Cultures of J774.2 macrophages were grown in minimum essential medium with glutamine $(2 \mathrm{mM})$ supplemented with $20 \%$ inactive FBS and maintained in a humidified atmosphere containing $95 \%$ air and $5 \% \mathrm{CO}_{2}$ at $37^{\circ} \mathrm{C}$. The cells were seeded at a density of $1 \times 10^{4}$ cells/well in 24 -well microplates 
(Nunc) with rounded coverslips on the bottom and cultured for two days. Subsequently, the cells were infected with the promastigote forms of $L$. infantum and $L$. braziliensis in vitro at a ratio of 10:1 for $24 \mathrm{~h}$. The nonphagocytosed parasites were removed by washing and the macrophages were then incubated with the drugs (at $1,10,25,50$ and $100 \mu \mathrm{M}$ ) for $72 \mathrm{~h}$ at $37^{\circ} \mathrm{C}$ in $5 \% \mathrm{CO}_{2}$.

Drug activity was determined by the number of amastigotes in treated and untreated cultures observed in methanol-fixed, Giemsa-stained preparations. The number of amastigotes was determined by analysing 200 host cells distributed in randomly chosen microscopic fields. The antileishmanial effects were expressed as $\mathrm{IC}_{50}$ values.

Axenic amastigote assay - Axenic amastigote forms of $L$. braziliensis and L. infantum were cultured as described by Moreno et al. (2011). The promastigotes were transformed into amastigotes by culturing for three days in M199 medium (Invitrogen, Leiden, The Netherlands) supplemented with $10 \%$ heat-inactivated foetal calf serum, $1 \mathrm{~g} / \mathrm{L} \beta$-alanine, $100 \mathrm{mg} / \mathrm{L} \mathrm{L}$-asparagine, $200 \mathrm{mg} / \mathrm{L}$ sucrose, $50 \mathrm{mg} / \mathrm{L}$ sodium pyruvate, $320 \mathrm{mg} / \mathrm{L}$ malic acid, $40 \mathrm{mg} / \mathrm{L}$ fumaric acid, $70 \mathrm{mg} / \mathrm{L}$ succinic acid, $200 \mathrm{mg} / \mathrm{L}$ $\alpha$-ketoglutaric acid, $300 \mathrm{mg} / \mathrm{L}$ citric acid, $1.1 \mathrm{~g} / \mathrm{L}$ sodium bicarbonate, $5 \mathrm{~g} / \mathrm{L}$ 2-[morpholino]ethanesulfonic acid, $0.4 \mathrm{mg} / \mathrm{L}$ hemin and $10 \mathrm{mg} / \mathrm{L}$ gentamicin at a $\mathrm{pH}$ of 5.4 at $37^{\circ} \mathrm{C}$. The effect of each compound against the axenic amastigote forms was tested for $48 \mathrm{~h}$ using a Neubauer haemocytometric chamber. The antileishmanial effects were expressed as $\mathrm{IC}_{50}$ values.

Infection assay - The J774.2 macrophage cells were grown for two days under the same conditions used in the amastigote assay and subsequently infected in vitro with the promastigote forms of L. infantum and L. braziliensis at a ratio of 10:1. The drugs $\left(\mathrm{IC}_{25}\right.$ concentrations) were added at the same time and the cells were incubated for $12 \mathrm{~h}$ at $37^{\circ} \mathrm{C}$ in an atmosphere containing $5 \% \mathrm{CO}_{2}$. The non-phagocytosed parasites and the drugs were removed by washing and the infected cultures were subsequently grown for 10 days in fresh medium. Fresh culture medium was added every $48 \mathrm{~h}$. The drug activity was determined based on the percentage of infected cells and the number of amastigotes per infected cell (in treated and untreated cultures) in methanol-fixed, Giemsa-stained preparations. The percentage of infected cells and the mean number of amastigotes per infected cell were determined by analysing 200 host cells distributed in randomly chosen microscopic fields.

Metabolite excretion - Cultures of $L$. infantum and $L$. braziliensis promastigotes (initial concentration $5 \times 10^{5}$ cells $/ \mathrm{mL}$ ) received $\mathrm{IC}_{25}$ concentrations of the compounds (except for the control cultures). After incubation for $96 \mathrm{~h}$ at $28^{\circ} \mathrm{C}$, the cells were centrifuged at $400 \mathrm{~g}$ for $10 \mathrm{~min}$. The supernatants were collected and the excreted metabolites were evaluated with ${ }^{1} \mathrm{H}$ NMR; the chemical displacements were expressed in parts per million using sodium 2,2-dimethyl-2-silapentane-5-sulphonate as the reference signal. The chemical displacements used to identify the respective metabolites were consistent with those described by Fernández-Becerra et al. (1997).
Ultrastructural changes - The parasites were cultured at a density of $5 \times 10^{5}$ cells $/ \mathrm{mL}$ in MTL medium containing drugs at the $\mathrm{IC}_{25}$ concentration. After $96 \mathrm{~h}$, those cultures were centrifuged at $400 \mathrm{~g}$ for $10 \mathrm{~min}$. The resulting cell pellets were washed in PBS, mixed with $2 \%$ (v/v) p-formaldehyde-glutaraldehyde in $0.05 \mathrm{M}$ cacodylate buffer ( $\mathrm{pH} 7.4)$ for $4 \mathrm{~h}$ at $4^{\circ} \mathrm{C}$ and prepared for TEM as described by González et al. (2005).

\section{RESULTS}

In vitro antileishmanial activity - The $\mathrm{IC}_{50}$ values that were registered $72 \mathrm{~h}$ after the exposure of the promastigote forms, axenic amastigote forms and intracellular amastigote forms of $L$. infantum and L. braziliensis to compounds 1-5 were determined and are shown in Table. The values for the reference drug Glucantime are also included for comparison.

The results displayed in Table show that the leishmanicidal activities of compounds 1-5 are similar to and, in most cases, higher than that found for Glucantime for both the extra and intracellular forms of $L$. infantum. The toxicity data are more revealing; all five compounds that were tested proved to be far less toxic to macrophages than the reference drug. Compounds 5 and 4 were 31 and 25-fold less toxic than Glucantime, respectively, and even the most toxic of the derivatives (compound 1) is 12 -fold less toxic than Glucantime. The highest selectivity index (SI) values were found for compounds 5, 4 and 3 ; these values exceeded those of the reference drugs by 36,36 , and 36 -fold in the case of 5, 34, 40 and 34 -fold for 4, and 21, 28 and 26-fold for 3 for the promastigote, axenic amastigote and intracellular amastigote forms of L. infantum, respectively.

Very similar conclusions can be drawn from the results for L. braziliensis, which are shown in Table. Compounds 5 and 4 again gave the best SI values in the three assays that were performed, with values exceeding those of the reference drugs by 37, 52 and 37-fold in the case of 5 and by 32, 24 and 22-fold for 4. Compound 2 also showed very good SI values, which were slightly higher than those for compound 4.

Different authors have claimed that a compound should have an SI value 20-fold higher than that of the reference to be considered to possess leishmanicidal properties (Nwaka \& Hudson 2006). This requirement is satisfied by compounds $2-5$ against both $L$. infantum and L. braziliensis. Compound 1 was not included in further studies due to its low SI values.

The effect of compounds 2-5 on the intracellular replication of the amastigote forms was determined. The macrophages were grown and infected with promastigotes in the stationary phase. The parasites invaded the cells and were converted to amastigotes within one day after infection and the rate of host-cell infection reached its maximum on day 10 (control experiment). The $\mathrm{IC}_{25}$ of each product was used as the test dosage in these assays and Glucantime was the reference drug. As shown in Fig. 2A, the addition of compounds $2-5$ to macrophage cells infected with $L$. infantum promastigotes caused a significant decrease in the infection rate with respect to the control and all of the compounds tested proved to be 
TABLE

In vitro activity, toxicity and selectivity index found for the reference drugs and the terpenoides derivatives on promastigote, axenic amastigote and amastigote forms of Leishmania spp

\begin{tabular}{|c|c|c|c|c|c|c|c|}
\hline \multicolumn{8}{|c|}{ Leishmania infantum } \\
\hline \multirow[b]{2}{*}{ Compounds } & \multicolumn{3}{|c|}{$\left(\mathrm{IC}_{50} \mu \mathrm{M}\right)$} & \multirow[b]{2}{*}{$\begin{array}{l}\text { Toxicity }\left(\mathrm{IC}_{50} \mu \mathrm{M}\right) \\
\quad \text { on } \mathrm{J} 774.2 \phi^{a}\end{array}$} & \multicolumn{3}{|c|}{ Selectivity index ${ }^{b}$} \\
\hline & Promatigote & $\begin{array}{c}\text { Axenic } \\
\text { amastigote }\end{array}$ & $\begin{array}{l}\text { Intracells } \\
\text { amastigote }\end{array}$ & & Promatigote & $\begin{array}{c}\text { Axenic } \\
\text { amastigote }\end{array}$ & $\begin{array}{l}\text { Intracells } \\
\text { amastigote }\end{array}$ \\
\hline Glucatime $^{\circledR}$ & $18.0 \pm 1.1$ & $30.0 \pm 3.4$ & $31.1 \pm 5.7$ & $15.2 \pm 1.0$ & 0.8 & 0.5 & 0.5 \\
\hline 1 & $17.8 \pm 3.3$ & $27.5 \pm 3.1$ & $26.3 \pm 3.9$ & $189.6 \pm 10.8$ & $11(14)$ & $7(14)$ & $7(14)$ \\
\hline 2 & $18.5 \pm 2.1$ & $18.9 \pm 2.1$ & $25.6 \pm 3.0$ & $257.6 \pm 16.1$ & $14(17)$ & $13(26)$ & $10(20)$ \\
\hline 3 & $17,7 \pm 0.4$ & $20.9 \pm 0.8$ & $22.3 \pm 2.6$ & $300.0 \pm 15.3$ & $17(21)$ & $14(28)$ & $13(26)$ \\
\hline 4 & $14.0 \pm 1.5$ & $19.2 \pm 1.3$ & $22.3 \pm 1.8$ & $379.0 \pm 9.9$ & $27(34)$ & $20(40)$ & $17(34)$ \\
\hline 5 & $16.1 \pm 2.4$ & $26.3 \pm 2.7$ & $26.6 \pm 6.3$ & $467.4 \pm 22.2$ & $29(36)$ & $18(36)$ & $18(36)$ \\
\hline \multicolumn{8}{|c|}{ Leishmania braziliensis } \\
\hline Glucatime $^{\circledR}$ & $25.6 \pm 1.6$ & $29.6 \pm 2.5$ & $28.3 \pm 4.3$ & $15.2 \pm 1.0$ & 0.6 & 0.5 & 0.6 \\
\hline 1 & $24.9 \pm 5.7$ & $40.1 \pm 2.7$ & $35.0 \pm 5.5$ & $189.6 \pm 10.8$ & $8(13)$ & $5(10)$ & $5(8)$ \\
\hline 2 & $13.0 \pm 1.5$ & $15.7 \pm 1.4$ & $18.0 \pm 2.8$ & $257.6 \pm 16.1$ & $20(33)$ & $16(32)$ & $14(23)$ \\
\hline 3 & $19.5 \pm 0.5$ & $30.9 \pm 2.8$ & $26.5 \pm 2.9$ & $300.0 \pm 15.3$ & $15(25)$ & $10(20)$ & $11(19)$ \\
\hline 4 & $19.6 \pm 1.1$ & $31.6 \pm 1.7$ & $29.0 \pm 4.0$ & $379.0 \pm 9.9$ & $19(32)$ & $12(24)$ & $13(22)$ \\
\hline 5 & $20.7 \pm 5.2$ & $18.0 \pm 0.8$ & $21.2 \pm 2.2$ & $467.4 \pm 22.2$ & $22(37)$ & $26(52)$ & $22(37)$ \\
\hline
\end{tabular}

$a$ : on $\mathrm{J} 774.2$ macrophages cells after $72 \mathrm{~h}$ of culture. $\mathrm{IC}_{50}=$ the concentration required to give $50 \%$ inhibition calculated by linear regression analysis from the $\mathrm{Kc}$ values at concentrations employed $(1,10,25,50$ and $100 \mu \mathrm{M}) ; b$ : selectivity index $(\mathrm{SI})=\mathrm{IC}_{50}$ vero cells $/ \mathrm{IC}_{50}$ promastigote, axenic amastigote, intracells amastigote forms. In brackets: number of times that compound SI exceeds the reference drug SI. Average of three separate determinations.

more effective than Glucantime. Compounds 5 and 2 (infectivity reduction of $93 \%$ and $85 \%$, respectively) were the most effective. Calculations of the average number of amastigotes per infected macrophage cell (Fig. 2C) supported these conclusions; all four compounds proved to be much more effective than Glucantime, which had only a $59 \%$ decrease in the number of amastigotes per infected macrophage.

The same experiment was performed with L. braziliensis and resulting infection rates and numbers of amastigotes per cell are presented in Fig. 2B, D, respectively. In both cases, all four compounds (2-5) were more effective than Glucantime and compounds 5 and 2 again proved to be the most active.

Studies on the mechanism of action - We performed several experiments to elucidate a possible mechanism of action for the abietane phenols 2-5 on the parasite.

Metabolite excretion effect - Leishmania species have a high rate of glucose consumption, which results in the acidification of the culture medium due to the incomplete oxidation of glucose. ${ }^{1} \mathrm{H}$ NMR spectroscopy enables the identification of the fermented metabolites excreted by the parasites during in vitro culture. Fig. 3A shows the spectrum produced by cell-free medium four days after inoculation with $L$. infantum. The peaks that correspond to the major metabolites produced and excreted during growth were apparent when this spectrum was compared with that of the fresh medium (spectra not shown). L. infantum excretes succinate and acetate as its major metabolites. The ${ }^{1} \mathrm{H}$ NMR spectra of the medium from drug-treated cells showed that only compound 5 significantly altered the metabolites excreted by $L$. infantum. When the promastigote forms of $L$. infantum were treated with compound 5 at $\mathrm{IC}_{25}$ doses, the excretion of the catabolites succinate and acetate was clearly altered (Fig. 2B) and a new peak, which was subsequently identified as pyruvate, appeared in the spectrum. All compounds exhibited similar behaviour towards $L$. braziliensis and compound 5 again appeared to have the largest inhibitory effect (spectra not shown).

Ultrastructural changes - TEM revealed substantial morphological alterations in Leishmania spp promastigotes after treatment with the newly synthesised abietane phenols 2-5 compared with the control sample (Fig. 4). All of the tested compounds induced alterations in $L$. infantum promastigotes, but only compounds 4 and 5 proved to affect $L$. braziliensis. The ultrastructural alterations induced by these compounds in the two Leishmania species can be seen in Fig. 4.

The derivative that was most effective against $L$. infantum was compound 4 (Fig. 4C), which induced the marked formation of vacuoles, some of which were completely empty, while others contained cellular debris. In some cases, these vacuoles occupied the entire cytoplasm and the parasites appeared swollen and deformed with swollen kinetoplasts and mitochondria. Some parasites 

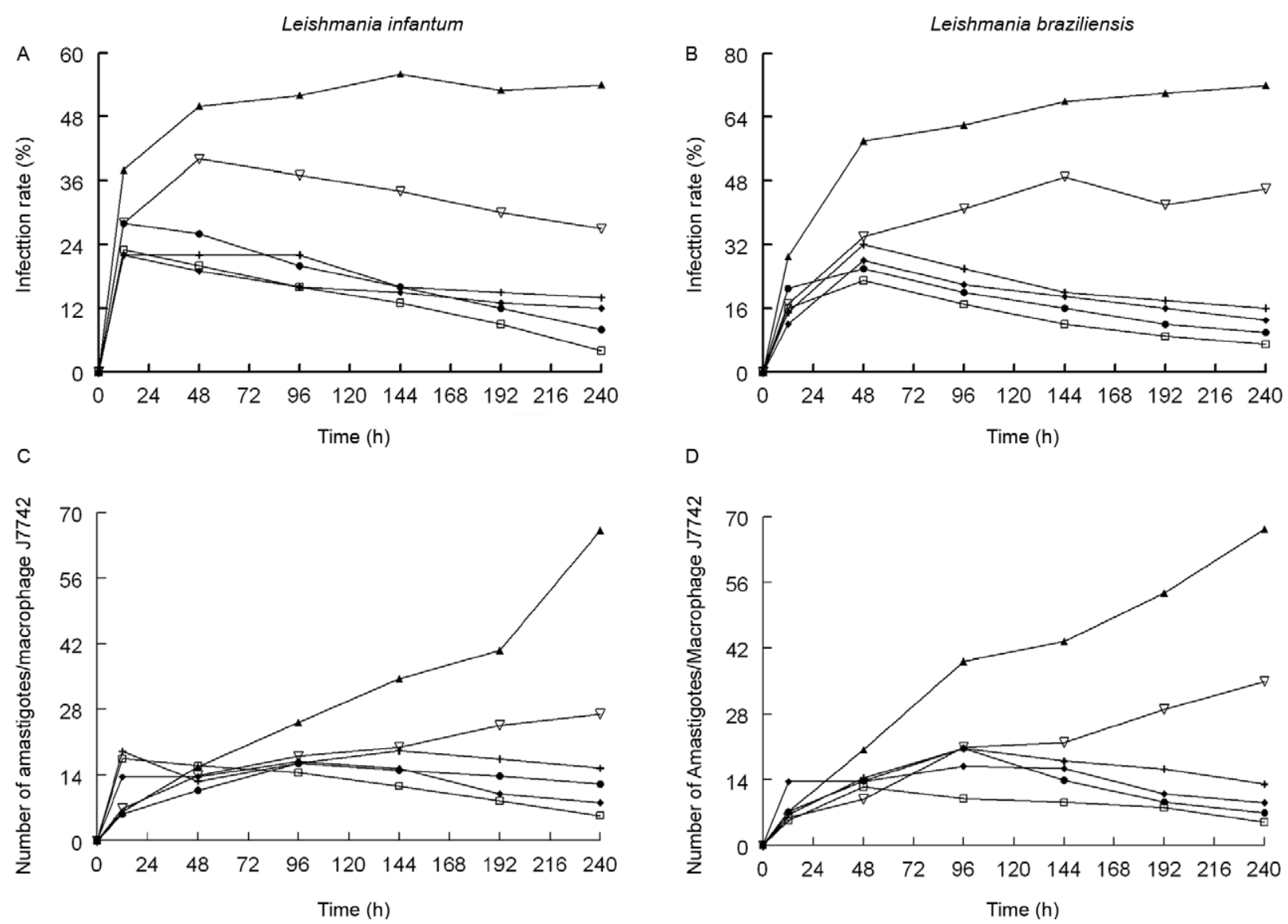

Fig. 2: effect of terpenoid derivatives on J774.2 macrophages infected with Leishmania infantum (A, C) and Leishmania braziliensis (B, D). Each value is the mean of three separate experiments. $\mathbf{\Delta}$ : control; $\nabla$ : Glucantime; $\bullet$ : compound 2 ; + compound 3 ; $\bullet$ : compound 4 ; $\square$ : compound 5 (at $\mathrm{IC}_{25}$ concentration).

were filled with lipid vacuoles. In addition, circular structures that were similar to glycosomes, but were strongly electrodense and may have contained metabolic products of the parasites, were observed; these structures were not observed in the untreated parasites. Likewise, numerous promastigotes were found to be strongly electrodense and the cytoplasmic organelles could not be distinguished. Although the remaining compounds were less effective against $L$. infantum, they all induced the formation of large numbers of empty vacuoles or cellular debris that resulted from the destruction of the parasites (data not shown).

Compound 4 was also the most effective against $L$. braziliensis, as shown in panel 4 of Fig. 4. In this case, a high percentage of the parasites appeared to be deformed and to contain vacuoles, which were either empty or filled with membranous structures and occupied almost the entire cytoplasm. Many of the parasites were strongly electrodense and appeared to be dead.

\section{DISCUSSION}

Natural products from plants are frequently studied by researchers in attempts to identify bioactive substances that could be useful against various diseases (Braña et al. 2005). The isolation and structural elucidation of sulphated glucoside and seco-A-ring oleanane-type triterpenoids has recently been described and the antileishmanial activity of these compounds has been examined. The results showed that the acylated seco-A ring oleanane derivatives are active, while the tricarboxylic acids are inactive (Macahig et al. 2011).

PX-6518 (1,10 13,28-epoxy-oleanane triterpene saponin) possesses potent in vivo activity against cutaneous (Inocêncio da Luz et al. 2011) and visceral (Maes et al. 2004) Leishmania species.

A previous study indicated that the newly synthesised abietane phenols 1-5 are prospective chemotherapeutic drugs for the treatment of diseases caused by Trypanosoma cruzi (unpublished observations). Most studies on the in vitro biological activity of new compounds against Leishmania spp are performed on the promastigote forms because they are much easier to work with in vitro. However, because extracellular forms are not the developed forms of the parasite in vertebrate hosts, studies using the extracellular forms are merely indicative of the potential leishmanicidal activity of the tested compounds. Consequently, a preliminary test using extracellular promastigote forms should always be complemented by a subsequent evaluation using intracellular forms (amastigotes in vertebrate host cells) to better evaluate the true leishmanicidal activity of the tested compounds 
(González et al. 2005). Therefore, we chose to study the activity on both intra and extracellular forms.

The inhibitory effect of abietane compounds on promastigote forms has been studied previously. Totarol, ferruginol and 7 $\beta$-hydroxyabieta-8,13-diene-11,12-dione have shown potent antileishmanial activity $\left(\mathrm{IC}_{50}\right.$ values of $3.5-4.6 \mu \mathrm{g} / \mathrm{mL}$ vs. $1.3 \mu \mathrm{g} / \mathrm{mL}$ for pentamidine) against Leishmania donovani promastigotes (Samoylenko et al. 2008). Abietane derivatives have also shown appreciable in vitro antileishmanial activity against the intracellular
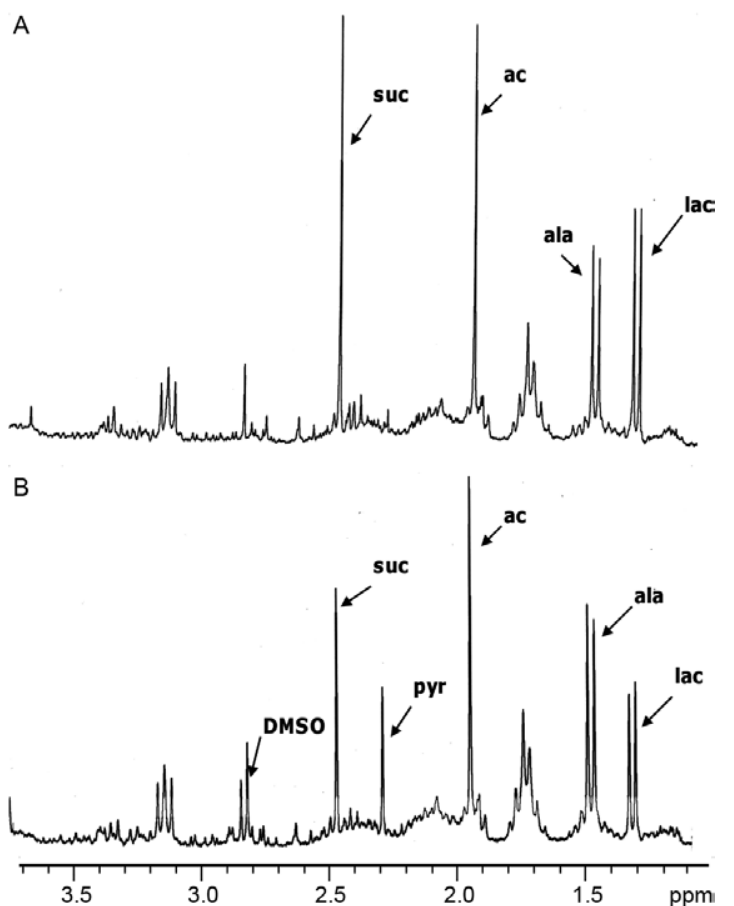

Fig. 3: ${ }^{1} \mathrm{H}$ nuclear magnetic resonance spectroscopy spectra of promastigote forms of Leishmania infantum. Control (A) and treated (B) with compound 5 at the concentration of $\mathrm{IC}_{25}(8 \mu \mathrm{M})$ ac: acetate [singlet, 1.94 parts per million (ppm)]; ala: L-alanine (doublet, $1.5 \mathrm{ppm}, 7.25 \mathrm{~Hz}$ ); DMSO: dimethyl sulfoxide; lac: lactate (doublet, 1.35 ppm, $6.84 \mathrm{~Hz}$ ); pyr: pyruvate (singlet, $2.26 \mathrm{ppm}$ ); suc: succinate: (singlet, $2.43 \mathrm{ppm}$ ). amastigote forms of L. donovani and Leishmania major in other studies (Tan et al. 2002).

Our results indicate that the leishmanicidal activity of these compounds against both the extra and intracellular forms of $L$. infantum and L. braziliensis is similar to that of the reference drug Glucantime, but they are less toxic to host cells. Furthermore, the infection rate decreased significantly upon treatment with these drugs. To the best of our knowledge, none of the previously studied trypanosomatids is capable of completely degrading glucose to $\mathrm{CO}_{2}$ under aerobic conditions, which means that they excrete a large proportion of their carbon skeleton into the medium as fermented metabolites, the profile of which differs according to the species (Bringaud et al. 2006).

Similar to T. cruzi (Ginger 2005), Leishmania spp excretes succinate as one of its major metabolites. The primary role of this metabolite is likely to maintain the glycosomal redox balance by providing two glycosomal oxidoreductase enzymes. These enzymes allow the reoxidation of NADH, which is produced by glyceraldehyde3 -phosphate dehydrogenase in the glycolytic pathway. Succinic fermentation offers one significant advantage in that it requires only half of the phosphoenolpyruvate (PEP) produced to maintain the $\mathrm{NAD}^{+} / \mathrm{NADH}$ balance. The remaining PEP may be converted into acetate, depending on the species.

The metabolite profile observed when the parasites were cultured in the absence of compounds 2-5 was consistent with the findings of other authors (Ginger 2005). The new peak that appeared when the promastigote forms were treated with compound 5 at $\mathrm{IC}_{25}$ doses, which was subsequently identified as pyruvate, showed that compound 5 inhibits the glycosomal enzymes and causes pyruvate to be excreted as a final metabolite.

The sesquiterpene polygodial, which was isolated from stem barks of Drimys brasiliensis, affected the Leishmania mitochondria (Corrêa et al. 2011).

The ultrastructural alterations induced by the terpenoid-based products studied here, especially compound 4, on extracellular forms of L. infantum and L. braziliensis can explain the changes observed in the metabolic studies, primarily their effect on the cytoplasm, kinetoplasts and mitochondria.
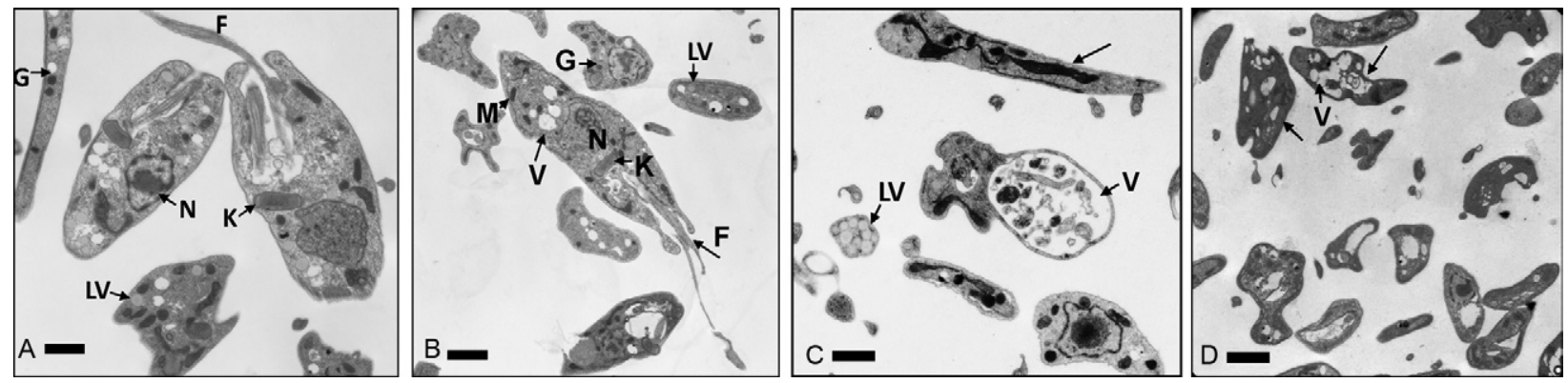

Fig. 4: transmission electron microscopy images of the ultrastructural alterations induced in Leishmania infantum and Leishmania braziliensis upon treatment with terpenoid derivatives. A: control parasite of $L$. infantum showing organelles with their characteristic aspect $(B a r=7.000)$; B: control parasite of L. braziliensis $(\mathrm{Bar}=4.400)$ : C: L. infantum treated with compound 4 showing intense vacuolisation and abundant electrodense structures (arrow) $(\mathrm{Bar}=7.000)$; D: L. braziliensis treated with compound 4. Some parasites are more electrodense (arrow), deformed and with intense vacuolisation $(\mathrm{Bar}=4.400)$; F: flagellum; G: glycosomes; K: kinetoplast; LV: lipid vacuoles; M: mitochondrion; N: nucleus. 
Thus, these in vitro results show that terpenoid compounds are potentially promising agents for the treatment of Leishmania infection. Further in vivo studies are warranted to further evaluate this potential.

\section{ACKNOWLEDGEMENTS}

To Encarnación Guerrero, for technical help in culture media preparation, to MJ Martínez-Guerrero and E OnoratoGutiérrez, for their expert technical help in transmission-electron microscopy and nuclear magnetic resonance spectroscopy, respectively.

\section{REFERENCES}

Alvarez-Manzaneda E, Chaboun R, Bentaleb F, Alvarez E, Escobar MA, Sad-Diki S, Cano MJ, Messouri I 2007a. Regioselective routes towards 14-hydroxyabietane diterpenes. A formal síntesis of immunosuppressant (-)- triptolide from (+)-abietic acid. Tetrahedron 63: 11204-11212.

Alvarez-Manzaneda E, Chahboun R, Cabrera E, Alvarez E, AlvarezManzaneda R, Lachkar M, Messouri I 2007b. First synthesis of picealactone $\mathrm{C}$. A new route toward taxodione-related terpenoids from abietic acid. Tetrahedron Lett 48: 989-992.

Alvarez-Manzaneda E, Chahboun R, Cabrera E, Alvarez E, AlvarezManzaneda R, Lachkar M, Messouri I 2007c. Synthesis of phenol abietane diterpenes based on the oxidative radical cyclization utilizing the $\mathrm{Mn}(\mathrm{OAc})_{3} / \mathrm{Ac}_{2} \mathrm{O}$ system. Synlett 15: 2425-2429.

Braña MF, Cacho M, García ML, Mayoral EP, López B, de Pascual-Teresa B, Ramos A, Acero N, Linares F, Muñoz-Mingarro D, Lozach O, Meijer L 2005. Pyrazolo[3,4c] pyridazines as novel and selective inhibitors of cyclindependent kinases. J Med Chem 48: 6843-6854.

Bringaud F, Riviere L, Coustou V 2006. Energy metabolism of trypanosomatids: adaptation to available carbon sources. Mol Biochem Parasitol 149: 1-9.

Cerecetto H, Gonzalez M 2002. Chemotherapy of Chagas' disease: status and new development. Curr Top Med Chem 2: 1187-1213.

Corrêa DS, Tempone AG, Reimão JQ, Taniwaki NN, Romoff P, Fávero OA, Sartorelli P, Mecchi MC, Lago JH 2011. Anti-leishmanial and anti-trypanosomal potential of polygodial isolated from stem barks of Drimys brasiliensis Miers (Winteraceae). Parasitol Res 109: 231-236.

Cui B, Zheng BL, He K, Zheng QY 2003. Imidazole alkaloids from Lepidium meyenii. J Nat Prod 66: 1101-1103.

Docampo R, Moreno SN 1986. Free radical metabolism of antiparasitic agents. Fed Proc 45: 2471-2476.

Fernández-Becerra C, Sánchez-Moreno M, Osuna A, Opperdoes FR 1997. Comparative aspects of energy metabolism in plant trypanosomatids. J Eukaryotic Microbiol 44: 523-529.

Ginger M 2005. Trypanosomatid biology and euglenozoan evolution: new insights and shifting paradigms revealed through genome sequencing. Protist 156: 377-392.

González P, Marín C, Rodríguez-González I, Hitos AB, Rosales MJ, Reina M, Diaz JG, Gonzalez-Coloma A, Sanchez-Moreno M 2005. In vitro activity of C20-diterpenoid alkaloid derivatives in promastigotes and intracellular amastigotes of Leishmania infantum. Int J Antimicrob Agents 25: 136-141.

Inocêncio da Luz RA, Vermeersch M, Deschacht M, Hendrickx S, Van Assche T, Cos P, Maes L 2011. In vitro and in vivo prophylactic and curative activity of the triterpene saponin PX-6518 against cutaneous Leishmania species. J Antimicrob Chemother 66: 350-353.

Kinouchi Y, Ohtsu H, Tokuda H, Nishino H, Matsunaga S, Tanaka R 2000. Potential antitumor-promoting diterpenoids from the stem bark of Piceaglehni. J Nat Prod 63: 817-820.
Luque F, Fernández-Ramos C, Entrala E, Rosales MJ, Navarro JA, Romero MA, Salas-Peregrín JM, Sánchez-Moreno M 2000. In vitro evaluation of newly synthesised $[1,2,4]$ triazolo[1,5-a]pyrimidine derivatives against Trypanosoma cruzi, Leishmania donovani and Phytomonas staheli. Comp Biochem Physiol 126: $39-44$.

Macahig RA, Matsunami K, Otsuka H 2011. Chemical studies on an endemic Philippine plant: sulfated glucoside and seco-A-ring triterpenoids from Dillenia philippinensis. Chem Pharm Bull 59: 397-401.

Maes L, Vanden Berghe D, Germonprez N, Quirijnen L, Cos P, De Kimpe N, Van Puyvelde L 2004. In vitro and in vivo activities of a triterpenoid saponin extract (PX-6518) from the plant Maesa balansae against visceral Leishmania species. Antimicrob Agents Chemother 48: 130-136.

Marrero JG, Andres LS, Luis JG 2002. Semisynthesis of rosmanol and its derivatives. Easy access to abietatriene diterpenes isolated from the genus Salvia with biological activities. J Nat Prod 65: 986-989.

Martin D, Tholl D, Gershenzon J, Bohlmann J 2002. Methyl jasmonate induces traumatic resin ducts, terpenoid resin biosynthesis and terpenoid accumulation in developing xylem of Norway spruce stems. Plant Physiol 129: 1003-1018.

Momeni AZ, Reiszadae MR, Aminjavaheri M 2002. Treatment of cutaneous leishmaniasis with a combination of allopurinol and lowdose meglumine antimoniate. Int J Dermatol 41: 441-443.

Moreno D, Plano D, Baquedano Y, Jiménez-Ruiz A, Palop JA, Sanmartín C 2011. Antileishmanial activity of imidothiocarbamates and imidoselenocarbamates. Parasitol Res 108: 233-239.

Muhammad I, Zhao J, Dunbar DC, Khan IA 2002. Constituents of Lepidium meyenii 'maca'. Phytochemistry 59: 105-110.

Natera S, Machuca C, Padrón-Nieves M, Romero A, Díaz E, Ponte-Sucre A 2007. Leishmania spp: proficiency of drug-resistant parasites. Int J Antimicrob Agents 29: 637-642.

Nwaka S, Hudson A 2006. Innovative lead discovery strategies for tropical diseases. Nature Rev 5: 941-955.

Palumbo E 2009. Current treatment for cutaneous leishmaniasis: a review. Am J Ther 16: 178-182.

Samoylenko V, Dunbar DC, Gafur MA, Khan SI, Ross SA, Mossa JS, El-Feraly FS, Tekwani BL, Bosselaers J, Muhammad I 2008. Antiparasitic, nematicidal and antifouling constituents from $J u$ niperus berries. Phytother Res 22: 1570-1576.

San Feliciano A, Gordaliza M, Salinero, MA, Miguel del Corral JM 1993. Abietane acids: sources, biological activities and therapeutic uses. Planta Med 59: 485-490.

Son KH, Oh HM, Choi SK, Han DC, Kwon BM 2005. Anti-tumor abietane diterpenes from the cones of Sequoia sempervirens. Bioorg Med Chem Lett 15: 2019-2021.

Takahashi M, Fuchino H, Sekita S, Satake M 2004. In vitro leishmanicidal activity of some scarce natural products. Phytother Res 18: 573-578.

Tan N, Kaloga M, Radtke 0A, Kiderlen AF, Oksuz S, Ulubelen A, Kolodziej H 2002. Abietane diterpenoids and triterpenoic acids from Salvia cilicica and their antileishmanial activities. Phytochemistry 6: 881-884.

Tasdemir D, Kaiser M, Brun R, Yardley V, Schmidt TJ, Tosun F, Rüedi P 2006. Antitrypanosomal and antileishmanial activities of flavonoids and their analogues: in vitro, in vivo, structure-activity relationship and quantitative structure-activity relationship studies. Antimicrob Agents Chemother 50: 1352-1364. 